\title{
Chromospheric Condensation and Quasi-periodic Pulsations in a Circular-ribbon Flare
}

\author{
Q. M. Zhang ${ }^{1,2}$, D. Li $^{1}$, and Z. J. Ning ${ }^{1}$ \\ ${ }^{1}$ Key Laboratory for Dark Matter and Space Science, Purple Mountain Observatory, CAS, \\ Nanjing 210008, China \\ zhangqm@pmo.ac.cn \\ ${ }^{2}$ Key Laboratory of Solar Activity, National Astronomical Observatories, CAS, Beijing \\ 100012, China
}

Received __; accepted _

Submitted to ApJ 


\begin{abstract}
In this paper, we report our multiwavelength observations of the C3.1 circularribbon flare SOL2015-10-16T10:20 in active region (AR) 12434. The flare consisted of a circular flare ribbon (CFR), an inner flare ribbon (IFR) inside, and a pair of short parallel flare ribbons (PFRs). The PFRs located to the north of IFR were most striking in the Interface Region Imaging Spectrograph (IRIS) $1400 \AA$ and $2796 \AA$ images. For the first time, we observed the circular-ribbon flare in the Ca II H line of the Solar Optical Telescope (SOT) aboard Hinode, which has similar shape as observed in the Atmospheric Imaging Assembly (AIA) $1600 \AA$ aboard the Solar Dynamic Observatory (SDO). Photospheric line-of-sight magnetograms from the Helioseismic and Magnetic Imager (HMI) aboard SDO show that the flare was associated with positive polarities and a negative polarity inside. The IFR and CFR were cospatial with the negative polarity and positive polarities, implying the existence of a magnetic null point $(\boldsymbol{B}=\mathbf{0})$ and the dome-like spine-fan topology. During the impulsive phase of the flare, "two-step" raster observations of $I R I S$ with a cadence of $6 \mathrm{~s}$ and an exposure time of $2 \mathrm{~s}$ show plasma downflow at the CFR in the Si IV $\lambda 1402.77 \operatorname{line}(\log T \approx 4.8)$, suggesting chromospheric condensation. The downflow speeds first increased rapidly from a few $\mathrm{km} \mathrm{s}^{-1}$ to the peak values of $45-52 \mathrm{~km} \mathrm{~s}^{-1}$, before decreasing gradually to the initial levels. The decay timescales of condensation were 3-4 minutes, indicating ongoing magnetic reconnection. Interestingly, the downflow speeds are positively correlated with logarithm of the Si IV line intensity and time derivative of the GOES soft X-ray (SXR) flux in 1-8 $\AA$. The radio dynamic spectra are characterized by a type III radio burst associated with the flare, which implies that the chromospheric condensation was most probably driven by nonthermal electrons. Using an analytical expression and the peak Doppler velocity, we de-
\end{abstract}


rived the lower limit of energy flux of the precipitating electrons, i.e., $0.65 \times 10^{10}$ erg $\mathrm{cm}^{-2} \mathrm{~s}^{-1}$. The Si IV line intensity and SXR derivative show quasi-periodic pulsations with periods of 32-42 s, which are likely caused by intermittent nullpoint magnetic reconnections modulated by the fast wave propagating along the fan surface loops at a phase speed of $950-1250 \mathrm{~km} \mathrm{~s}^{-1}$. Periodic accelerations and precipitations of the electrons result in periodic heating observed in the $\mathrm{Si}$ IV line and SXR.

Subject headings: Sun: corona - Sun: chromosphere - Sun: flares — Sun: X-rays, gamma rays — techniques: spectroscopic 
Online-only material: animations, color figures

\section{Introduction}

Solar flares are one of the most spectacular activities in the solar system. Up to $10^{29}-10^{32}$ ergs magnetic free energies are impulsively released, accompanied by rapid increases of emissions in various wavelengths (Shibata \& Magara 2011). It is generally believed that magnetic reconnection plays a key role in the reconfiguration of magnetic field lines and conversion of magnetic energy into kinetic and thermal energies of plasma (Forbes \& Acton 1996; Priest \& Forbes 2000). In the context of standard solar flare model (Carmichael 1964; Sturrock 1966; Hiravama 1974; Kopp \& Pneuman 1976), the accelerated nonthermal electrons propagate downward and heat the chromosphere, forming the bright flare ribbons in $\mathrm{H} \alpha, \mathrm{Ca}$ II $\mathrm{H}$, ultraviolet (UV), and extreme-ultraviolet (EUV) wavelengths. The flare ribbons have diverse shapes, with two parallel flare ribbons (PFRs) being the most commonplace (Li \& Zhang 2015). Sometimes, circular flare ribbons (CFRs) appear accompanied by remote brightenings, which is generally believed to be associated with a magnetic null point $(\boldsymbol{B}=\mathbf{0})$ and the dome-like spine-fan topology (Masson et al. 2009; Zhang et al. 2012; Pontin et al. 2016). More complex flare ribbons have also been observed (Joshi et al. 2015). Apart from the localized heating, hard X-ray (HXR) emissions are generated via Coulomb collisions (Brown 1971). If open magnetic field lines are involved, it is likely that the nonthermal electrons at speeds of $0.06-0.25 c$ ( $c$ is the speed of light) escape the Sun into the interplanetary space and generate type III radio bursts with frequency ranging from 0.2 to hundreds of MHz (Dulk et al. 1987; Aschwanden et al. 1995; Zhang et al. 2015). Sometimes, the HXR and radio emissions of a flare show quasi-periodic pulsations $(\mathrm{QPPS})$, with their periods ranging from milliseconds (Tan et al. 2010) through a few seconds (Kliem et al. 2000; Asai et al. 2001; Ning et al. 
2005; Nakariakov et al. 2010; Haves et al. 2016) to several minutes (Ofman \& Sui 2006; Sych et al. 2009; Nakariakov \& Melnikov 2009; Ning 2014). So far, QPPs have been extensively explored using both imaging ( $\mathrm{Su}$ et al. 2012a, b) and spectral observations (Mariska 2005). Li et al. (2015b) studied the X1.6 flare on 2014 September 10. Four-minute QPPs are evident not only in the HXR, EUV, UV, and radio light curves, but also in the temporal evolutions of the Doppler velocities and line widths of the $\mathrm{C}$ I, O IV, Si IV, and Fe Xxi lines. Brosius \& Daw (2015) studied the M7.3 flare on 2014 April 18. The chromospheric and transition region line emissions show quasi-periodic intensity and velocity fluctuations with periods of $\sim 3$ minutes during the first four peaks and $\sim 1.5$ minutes during the last four peaks.

The overpressure of the chromosphere drives evaporation of hot and dense plasma upward into the newly reconnected coronal loops at speeds of tens to hundreds of $\mathrm{km}$ $\mathrm{s}^{-1}$ (Fisher et al. 1985a, $\left., \mathrm{b}, \mathrm{c}\right)$. At the same time, downward chromospheric condensation takes place at speeds of a few to tens of $\mathrm{km} \mathrm{s}^{-1}$ as a result of the balance of momentum (Teriaca et al. 2003). In the past 40 years, the investigations of chromospheric evaporation and condensation have greatly benefitted from the spectroscopic observations in $\mathrm{H} \alpha$ and EUV wavelengths from the ground-based as well as space-borne telescopes (e.g., Czavkowska et al. 1999; Brosius \& Phillips 2004; Chen \& Ding 2010; Li \& Ding 2011; Milligan 2011; Young et al. 2013; Tian et al. 2014, 2015; Li et al. 2015a; Polito et al. 2015, 2016; Battaglia et al. 2015; Graham \& Cauzzi 2015). Ichimoto \& Kurokawa (1984) studied the red asymmetry of $\mathrm{H} \alpha$ line profiles of 4 flares near the disk center. The first two on June 20 and June 21 in 1982 were indeed circular-ribbon flares. The red-shifted emission streaks of $\mathrm{H} \alpha$ line are caused by the conspicuous downward motion in the flare chromospheric region with velocities of $40-100 \mathrm{~km} \mathrm{~s}^{-1}$. Using the spectral data of an M6.8 two-ribbon flare observed by the Coronal Diagnostic Spectrometer (CDS) aboard SOHO, Czaykowska et al. (1999) found that the Fe XVI downward velocity of the regions between 
the flare ribbons and the magnetic neutral line decreases from $\sim 90 \mathrm{~km} \mathrm{~s}^{-1}$ to $\sim 15 \mathrm{~km}$ $\mathrm{s}^{-1}$ within 2.5 minutes in the gradual phase, indicating ongoing magnetic reconnection. Brosius \& Phillips (2004) studied an M2.0 flare observed by CDS and found that the downward velocity of chromospheric condensation in $\mathrm{O} v$ line decreases from $\sim 40 \mathrm{~km}$ $\mathrm{s}^{-1}$ to zero within 3 minutes. Compared with CDS, the Extreme-ultraviolet Imaging Spectrometer (EIS; Culhane et al. 2007) aboard Hinode (Kosugi et al. 2007) covers a much wider temperature range and has much higher resolution and cadence. Using the spectral data of a C1.0 flare observed by EIS, Brosius (2013) found that the upward velocities of chromospheric evaporation in Fe XXIII line decreases from its maximum value $(>200$ $\mathrm{km} \mathrm{s}^{-1}$ ) to zero within 2 minutes. The successful launch of the Interface Region Imaging Spectrograph (IRIS; De Pontieu et al. 2014) opened a new era of flare research, thanks to its unprecedented resolution and cadence. The timescales of chromospheric evaporation range from 5 to 12 minutes in most cases (Battaglia et al. 2015; Li et al. 2015a; Tian et al. 2015; Polito et al. 2015, 2016; Graham \& Cauzzi 2015). However, it can reach $\sim 20$ minutes in an M1.0 flare (Sadvkov et al.|2016). The timescales of chromospheric condensation range from 1 to 7 minutes (Graham \& Cauzzi 2015; Sadykov et al. 2016). A delay of $>60$ s between the starting times of condensation and evaporation has been observed (Graham \& Cauzzi 2015; Young et al. 2015). Both the upward evaporation velocities and downward condensation velocities are positively correlated with the HXR fluxes, which is consistent with the numerical model of evaporation driven by nonthermal electrons (Tian et al. 2015; Li et al. 2015a).

So far, chromospheric condensation in circular-ribbon flares has rarely been investigated. Using the imaging and raster observations of a GOES C4.2 circular-ribbon flare on 2015 October 16 by IRIS, Zhang et al. (2016) found explosive chromospheric evaporation during the impulsive phase of the flare, which is characterized by simultaneous plasma upflow $\left(35-120 \mathrm{~km} \mathrm{~s}^{-1}\right)$ observed in the high-temperature Fe Xxi $\lambda 1354.09 \operatorname{line}(\log T \approx 7.05)$ and 
downflow $\left(10-60 \mathrm{~km} \mathrm{~s}^{-1}\right)$ observed in the low-temperature Si IV $\lambda 1393.77 \operatorname{line}(\log T \approx 4.8)$. Based on the quantitative estimation of nonthermal energy flux under the thick-target model and the fact that the inner flare ribbon (IFR) where chromospheric evaporation occurred was cospatial with the single HXR source at $12-25 \mathrm{keV}$, the authors concluded that the explosive chromospheric evaporation was most likely driven by nonthermal electrons accelerated by magnetic reconnection. However, the IRIS observation was in the "sparse synoptic raster" mode with a cadence of $9.4 \times 36=338.4 \mathrm{~s}$, which is difficult to study the temporal evolution of the same position. There were a couple of homologous circular-ribbon flares in the same active region (AR 12434) on the same day as the C4.2 flare. In this paper, we study another one, the C3.1 flare observed by IRIS. The raster observation was in the "two-step raster" mode with a cadence of $6 \mathrm{~s}$, which is quite suitable for investigating the temporal evolution of the same position of flare ribbon. In addition, we observed QPPs during the impulsive phase of the flare. In Section 2, we describe the instruments and data analysis using observations from various telescopes. Results and discussions are presented in Section 3 and Section 4, respectively. Finally, we draw a conclusion in Section 5 ,

\section{Instruments and data analysis}

\section{1. $\quad S D O$ and Hinode Observations}

The flare was observed by the Atmospheric Imaging Assembly (AIA; Lemen et al. 2012) aboard the Solar Dynamic Observatory (SDO) in the full-disk images. AIA has two UV $(1600 \AA, 1700 \AA)$ and 7 EUV filters, including $304 \AA$ ( $T \approx 0.05 \mathrm{MK}), 171 \AA(T \approx 0.63$ $\mathrm{MK}), 193 \AA(T \approx 1.5 \mathrm{MK}), 211 \AA(T \approx 2.0 \mathrm{MK}), 335 \AA(T \approx 2.5 \mathrm{MK}), 94 \AA(T \approx 6.3 \mathrm{MK})$, and $131 \AA$ ( $T \approx 0.37 \mathrm{MK}$ and $10 \mathrm{MK}$ ). Photospheric line-of-sight (LOS) magnetograms of the AR were observed by the Helioseismic and Magnetic Imager (HMI; Scherrer et al. 2012) aboard SDO. The level_1 data from AIA and HMI were calibrated using the standard 
Solar SoftWare (SSW) programs aia_prep.pro and hmi_prep.pro, respectively. It was also partially observed by the Ca II H ( 3968 $\AA$ ) filter of the Solar Optical Telescope (SOT; Tsuneta et al. 2008) aboard Hinode with a limited field of view (FOV) of $218^{\prime \prime} \times 109^{\prime \prime}$. SOT consists of 4 instruments, including the Broad-band Filter Imager (BFI), Narrow-band Filter Imager (NFI), Spectropolarimeter (SP), and Correlation Tracker (CT). BFI produces images in 6 bands (CN bands, Ca II H, G-band, and 3 continuum bands). The raw BFI data were calibrated using the SSW program fg_prep.pro. The magnetograms and images observed in Ca II H, UV, and EUV wavelengths were coaligned with an accuracy of $\sim 0^{\prime \prime} 6$.

\subsection{IRIS Observations}

The Slit-Jaw Imager (SJI) aboard IRIS observed the flare in Si IV $\lambda 1400(T \approx 0.065$ $\mathrm{MK})$ and $\mathrm{Mg}$ II $\mathrm{H} / \mathrm{K} \lambda 2796(T \approx 0.01 \mathrm{MK})$. The raster observation was in the "two-step raster" mode (OBS ID: 3660105413). As its name implies, each raster had two steps from east to west with a cadence of $6 \mathrm{~s}$ and an exposure time of $2 \mathrm{~s}$, covering an area of $2^{\prime \prime} \times 128^{\prime \prime} .42$. Each step had a spatial size of $0^{\prime \prime} 3327$ and a spectral scale of $\sim 25.44 \mathrm{~m} \AA$ pixel $^{-1}$, which equal to $\sim 5.44 \mathrm{~km} \mathrm{~s}^{-1}$ pixel $^{-1}$. The raster data of Si IV $\lambda 1402.77$ line $(\log T \approx 4.8)$ were preprocessed using the SSW programs iris_orbitvar_corr_l2.pro and iris_prep_despike.pro. Since the intensity of Fe xxi $\lambda 1354.09$ line was too weak to obtain convincing line profiles, we did not use this line. The Si IV line is an isolated line with red asymmetry. However, the spectra could not been simply fitted by a single-Gaussian or double-Gaussian function. To determine the line center $\left(\lambda_{c}\right)$ and intensity $(I)$, we use the same method as described in Brosius \& Daw (2015):

$$
\lambda_{c}=\frac{\int_{\lambda_{1}}^{\lambda_{2}} \lambda \times I_{\lambda} d \lambda}{\int_{\lambda_{1}}^{\lambda_{2}} I_{\lambda} d \lambda},
$$




$$
I=\int_{\lambda_{1}}^{\lambda_{2}} I_{\lambda} d \lambda,
$$

where $\lambda_{1}=1402.01 \AA$ and $\lambda_{2}=1403.61 \AA$ signify the lower and upper limits for the integrals. $I_{\lambda}$ denotes the intensity at $\lambda$.

\subsection{Radio Observations}

The flare was accompanied by a type III radio burst, which was recorded in the radio dynamic spectra by the WIND/WAVES (Bougeret et al. 1995) and two ground-based telescopes 1 (KRIM, BLENSW). WAVES has two detectors: RAD1 $(0.02-1.04 \mathrm{MHz})$ and RAD2 (1.075-13.825 MHz). The frequency ranges of KRIM and BLENSW are 250-350 $\mathrm{MHz}$ and $10-80 \mathrm{MHz}$, respectively. The observing parameters of the instruments are summarized in Table 1,

\section{Results}

\subsection{Flare Ribbons}

In Figure 1, the AIA $171 \AA$ image at 10:17:46 UT is displayed in panel (a). The C3.1 circular-ribbon flare with enhanced emission took place at the center of AR 12434. Panel (b) shows the HMI LOS magnetogram of the AR at 10:17:45 UT. In order to investigate the magnetic field of the flare, we give a close-up of the region within the dashed box in panel (d). As mentioned in Zhang et al. (2016), the flare region is characterized by a central negative polarity $(\mathrm{N})$ surrounded by positive polarities $(\mathrm{P})$. The contours of the $171 \AA$ intensity in panel (a) are superposed with red lines. It is clear that the flare

\footnotetext{
${ }^{1}$ http://e-callisto.org
} 
ribbons were cospatial with the strong magnetic field. Like the C4.2 flare, such a magnetic pattern is strongly indicative of a magnetic null point and the spine-fan configuration in the corona. The horizontal and vertical length scales of the flare are $L_{x} \approx 30^{\prime \prime}$ and $L_{y} \approx 40^{\prime \prime}$, respectively. Assuming a semispherical shape of the fan surface, the height of the null point and the loop length $(L)$ from the null point to the solar surface are estimated to be $0.5 \sqrt{L_{x} L_{y}}=17^{\prime \prime} .3$ and $\frac{\pi}{4} \sqrt{L_{x} L_{y}}=27^{\prime \prime} .2$, respectively. The height of null point is higher than that of the coronal bright points on 2007 March 16 (Zhang et al.|2012), but slightly lower than that of the M6.7 flare on 2011 September 8 (Zhang et al. 2015). Panel (c) demonstrates the HMI white-light continuum image at 10:17:45 UT, which is characterized by the dark sunspot and the surrounding plage of the AR.

Figure 2 shows the temporal evolution of the flare in $304 \AA$. Unlike the C4.2 flare during 13:36-13:51 UT, we did not find a preexisting filament residing in the AR and its eruption that would result in a blowout jet (Zhang et al. 2016). The flare started brightening from its southwest part at $\sim 10: 15$ UT (see panel (b)). As time goes on, the area of initial brightening expanded and became more complex, including several patches or kernels (see panels (d)-(e) and online movie anim304.mpg). Meanwhile, the narrow eastern part of the flare brightened, forming the CFR as indicated by the arrow in panel (e). After its peak time around 10:20 UT, the intensity of flare decreased. It is evident that the intensity of CFR decreased much faster than the IFR inside (see panels (g)-(i)).

In Figure 3, the top two rows demonstrate selected images of the flare in the other AIA wavelengths with higher formation temperatures (see online animation anim335.mpg). The evolution is similar to that in $304 \AA$. In panel (d), the contours of the positive and negative LOS magnetic fields are overlaid with magenta and orange lines. Like the C4.2 flare, the CFR was approximately cospatial with positive polarities, while the IFR was approximately cospatial with negative polarity (Zhang et al. 2016). The hot, bright post-flare loop (PFL) 
after being filled with evaporated material was obvious in $94 \AA$ (panel (e)) and $131 \AA$ (panel (f)), which is consistent with the peaks around 10:20 UT in the EUV light curves (Figure 6(a)). The bottom row shows the CFR and IFR observed in the lower solar atmosphere (1600 $\AA$, Ca II H, and $1700 \AA$ ). To our knowledge, this is the first report of a circular-ribbon flare observed in Ca II H. The shape of ribbon is similar to that in $1600 \AA$, but has finer structure owing to the higher resolution of SOT.

Figure 4 shows the selected $1400 \AA$ images during 10:15:15-10:36:10 UT (see online movie anim1400.mpg). The evolution of the flare is somewhat similar to that in $304 \AA$ in Figure 2, featuring the bright CFR and IFR with ultrafine structures (see panel (c)). Despite that the two filters have comparable formation temperatures, new features appear thanks to the high resolution of IRIS. In panel (c), a pair of bright PFRs are located to the north of IFR. The PFRs are $18^{\prime \prime} 4$ in length, which is much shorter than the CFR. The brightness of PFRs reached the maximum around 10:17:46 UT before decreasing gradually. Such a pattern, including the coexisting CFR, IFR, and PFRs, was also observed in the large-scale M7.3 flare on 2014 April 18 (Joshi et al. 2015). In panel (a), the slit positions of the two-step raster are drawn with vertical dashed lines (S1 and S2). In panel (d), S1 encounters the CFR at $\mathrm{H} 1$ and $\mathrm{H} 2$. S2 encounters the CFR at H1* and $\mathrm{H} 2 *$. The intensity of $\mathrm{H}^{*}$ is greater than $\mathrm{H} 1$, but lower than $\mathrm{H} 2$ and $\mathrm{H}^{*}$ in most cases.

Figure 5 shows the selected $2796 \AA$ images. The evolution of the flare is similar to that in $1400 \AA$, featuring the CFR, IFR, and PFRs (see panel (c)). However, the duration of brightening of the ribbons is much shorter owing to the much lower formation temperature of $2796 \AA(T \approx 0.01 \mathrm{MK})$.

In Figure 6, the upper panel shows the normalized light curves of the flare in EUV wavelengths. It is obvious that all the light curves experienced a sharp peak around 10:17:46 UT simulantously. For the light curve in $131 \AA$, the small peak around 10:17:46 UT 
indicates the existence of hot material up to $10 \mathrm{MK}$ during the early-impulsive phase of the flare (Fletcher et al. 2013; Tian et al. 2015). This is also supported by the "step" after the fast rise in the $94 \AA$ light curve. As the chromospheric evaporation proceeds possibly due to the nonthermal electrons that impact the chromosphere, the coronal loops are filled with hot and dense plasmas, which generate strong emissions in the wavelengths with the highest formation temperatures. This is revealed by the dominant peak at $\sim 10: 20: 10$ UT in $131 \AA$ and the following peak in $94 \AA$. As time goes on, the coronal loops cool down primarily due to thermal conduction and create peaks of emissions progressively at decreasing formation temperatures of $335 \AA, 211 \AA, 193 \AA, 171 \AA$, and $304 \AA$. The cooling timescale of the flare loops from $\sim 10 \mathrm{MK}$ to $\sim 0.6 \mathrm{MK}$ was $\sim 5$ minutes. The lower panel of Figure [6 shows the soft X-ray (SXR) light curves during 10:10-10:30 UT in 0.5-4 $\AA$ (cyan) and 1-8 $\AA$ (magenta). The flare had a short lifetime of $\sim 15$ minutes. It started at $\sim 10: 15$ UT, peaked at $\sim 10: 20 \mathrm{UT}$, and ended at $\sim 10: 30 \mathrm{UT}$.

\subsection{Chromospheric Condensation at the CFR}

In Figure 7, the IRIS spectral window of Si IV for S1 and S2 are displayed in the left and right panels, respectively. In the top two panels, the white curves represent the spacetime average spectra between the vertical positions $\left(-270^{\prime \prime}\right.$ and $\left.-230^{\prime \prime}\right)$ marked by the two yellow lines during 10:15:21-10:15:43 UT. Despite in the very initial phase of the flare, the spectra show weak red asymmetry. Hence, a single-Gaussian fitting is still inapplicable. Using Equation (11) and the spacetime average spectra, the rest wavelength $\left(\lambda_{0}\right)$ of $\mathrm{Si}$ IV

$\lambda 1402.77$ is calculated to be $1402.86 \pm 0.0145 \AA$, exactly the same value as in Brosius \& Daw (2015). In panels (c)-(h), the green curves are the spectra at $\mathrm{H} 1$ and $\mathrm{H} 1 *$ in three raster scans. The blue curves are the spectra at $\mathrm{H} 2$ and $\mathrm{H}_{2} *$ in three raster scans. For H1, the line profiles seem to be slightly blue-shifted, indicating weak plasma upflow. For H1*, the 
profiles are wider than $\mathrm{H} 1$ and show red asymmetry, implying plasma downflow. For H2 and $\mathrm{H}_{2}^{*}$, the red asymmetry are much more significant, suggesting faster downflow. The line centers and intensities at each position along S1 and S2 during the raster are calculated using Equations (1) and (2). The Doppler velocity $\left(V_{D}\right)$ of Si IV line is calculated based on the distances between line centers and $\lambda_{0}$ :

$$
V_{D}=\frac{c}{\lambda_{0}}\left(\lambda_{c}-\lambda_{0}\right)
$$

According to the uncertainty of $\lambda_{0}$, the uncertainty of $V_{D}$ is calculated to be $3.11 \mathrm{~km} \mathrm{~s}^{-1}$.

In Figure 8, the temporal evolutions of the Si IV line intensity and Doppler velocity along slit S1 are demonstrated in the left panels. The temporal evolutions of the intensity and Doppler velocity of $\mathrm{H} 1$ and $\mathrm{H} 2$ are extracted and plotted in the right panels with cyan and magenta lines. It is clear that outside the CFR, the intensities and $V_{D}$ are far less than the CFR. The intensities of H1 and H2 increased from 10:15:15 UT and reached the peak values before decreasing with oscillations. The intensity of $\mathrm{H} 2$ was greater than $\mathrm{H} 1$ in most cases during the flare. The red-shifted $V_{D}$ of $\mathrm{H} 2$ increased quickly and reached the maximum of $\sim 45 \mathrm{~km} \mathrm{~s}^{-1}$ at 10:16:24 UT before decreasing gradually to $\sim 2 \mathrm{~km} \mathrm{~s}^{-1}$ at 10:20:29 UT. The timescale of chromospheric condensation of $\mathrm{H} 2$ was $\sim 4$ minutes. The $V_{D}$ of $\mathrm{H} 1$ was blue-shifted and fluctuated slightly around $-5 \mathrm{~km} \mathrm{~s}^{-1}$ during most of the lifetime. The possible cause of coexisting downflow and upflow along the CFR will be discussed at the end of Section 4 .

In Figure 9, the temporal evolutions of the Si IV line intensity and Doppler velocity along slit S2 are demonstrated in the left panels. The temporal evolutions of the intensity and Doppler velocity of $\mathrm{H}^{*}$ and $\mathrm{H}^{*}$ are extracted and plotted in the right panels with cyan and magenta lines. The evolutions of intensities of $\mathrm{H} 1^{*}$ and $\mathrm{H} 2 *$ are similar to $\mathrm{H} 1$ and H2. The red-shifted $V_{D}$ of $\mathrm{H}_{2}^{*}$ increased quickly from $\sim 10 \mathrm{~km} \mathrm{~s}^{-1}$ at 10:15:18 UT and reached the maximum of $\sim 52 \mathrm{~km} \mathrm{~s}^{-1}$ at 10:17:09 UT before decreasing gradually to 
$\sim 10 \mathrm{~km} \mathrm{~s}^{-1}$ at 10:20:32 UT. The timescale of chromospheric condensation of $\mathrm{H}^{*}$ was $\sim 3.5$ minutes. The $V_{D}$ of $\mathrm{H}^{*}$ was red-shifted and fluctuated slightly around $4 \mathrm{~km} \mathrm{~s}^{-1}$ during most of the lifetime.

As mentioned above, the $\mathrm{Si}$ IV line intensities and $V_{D}$ of the CFR have similar trends, implying a temporal correlation between the two parameters. In Figure 10, the scatter plots of intensity and $V_{D}$ are drawn in the left panels for $\mathrm{H} 2$ and $\mathrm{H} 2 *$. Note that the intensities are in log-scale. It is obvious that the two parameters are positively correlated with correlation coefficients $\geq 0.8$.

\subsection{QPPs of the Flare}

In Figure $6(\mathrm{~b})$, the time derivative of SXR flux in $1-8 \AA$ is plotted with a red line, which is characterized by several regular peaks in the impulsive phase. Using the same method as described in Li et al. (2015b), we decompose the SXR derivative into a slow-varying component plus a rapid-varying component, the later of which is displayed in Figure 11(a). It is clearly revealed that the SXR derivative fluctuated quasi-periodically. The Morlet wavelet transform of the fast-varying component is illustrated in Figure 11(b). The quasi-periodicity, i.e., QPPs during 10:16:20-10:21:10 UT, is confirmed. The periods are 25-40 s with its maximum power lying at $\sim 32 \mathrm{~s}$.

In Figure 8 and Figure 9, the violent fluctuations of the Si IV intensities of the CFR imply the existence of QPPs in UV wavelengths. In order to justify the conjecture, we decompose the light curves in Figure 8(b) and Figure 9(b) into slow-varying components and fast-varying components by smoothing. The Morlet wavelet transforms of the fast-varying components are illustrated in Figure 12. The QPPs in UV wavelengths during 10:16:05-10:19:05 UT are confirmed. In the left panels, the periods range from 25 to 60 
$\mathrm{s}$, with the maximum power lying at $\sim 42 \mathrm{~s}$ for $\mathrm{H} 1$ and $\sim 38$ s for $\mathrm{H} 2$. In the right panels, the periods range from 25 to $50 \mathrm{~s}$, with the maximum power lying at $\sim 35 \mathrm{~s}$ for $\mathrm{H}^{*}$ and $\sim 33 \mathrm{~s}$ for $\mathrm{H}^{*}$. It is evident that the periods are very close and slightly longer than the period in SXR (32 s). The evolutions of $V_{D}$ in Figure 8(d) and Figure 9(d), however, do not show QPPs. In Figure 6(b), the light curve of the flare in $1400 \AA$ is drawn with a blue line. By and large, the total $1400 \AA$ intensity, as a signature of heating at the chromosphere by nonthermal electrons, has similar evolution to the SXR derivative. Correlation analysis shows that the two parameters are temporally correlated (c.c. $\approx 0.77$ ) during the impulsive phase. However, QPP was absent in the $1400 \AA$ light curve.

\section{Discussion}

\subsection{What Is the Cause of Chromospheric Condensation?}

The observational studies of chromospheric evaporation and condensation have a long history. Ichimoto \& Kurokawa (1984) studied the temporal evolution of the H $\alpha$ line profiles of 4 flares near the disk center. The timescales of the total evolutions are $\sim 1$ minute. The authors proposed that the red asymmetry of $\mathrm{H} \alpha$ line results from the downward motion of the compressed chromospheric region produced by the impulsive heating by energetic electron beam or thermal conduction. Li et al. (2015a) found positive correlations between the HXR emissions and upward Doppler shifts of Fe xxi line and downward Doppler shifts of $\mathrm{C}$ I line $(\log T \approx 4.0)$ in two X1.6 flares. In the C4.2 circular-ribbon flare, Zhang et al. (2016) found that the IFR where explosive chromospheric evaporation occurred was cospatial with the single HXR source. A quantitative estimation of the energy flux of the nonthermal electron supports the electron-driven evaporation/condensation.

Like in Li et al. (2015a), we explored the relationship between the downward $V_{D}$ of the 
CFR and SXR derivative for the C3.1 flare. The scatter plots of the two parameters are drawn in the right panels of Figure 10 for $\mathrm{H} 2$ and $\mathrm{H} 2^{*}$, indicating that they are positively correlated with correlation coefficients $\geq 0.7$. In Figure 13 , the radio dynamic spectra from KRIM and BLENSW are displayed in the top two panels. The most striking feature is the type III burst with enhanced emissions and rapid frequency drift during 10:17:00-10:18:00 UT. The dynamic spectra from RAD1 and RAD2 aboard WIND/WAVES are displayed in the bottom two panels. The frequency of burst drifted rapidly from $13 \mathrm{MHz}$ at 10:18 UT to $1 \mathrm{MHz}$ at 10:20 UT and then drifted slowly to $\sim 0.2 \mathrm{MHz}$ until $\sim 10: 40 \mathrm{UT}$. The radio flux at $42.5 \mathrm{MHz}$ is plotted in Figure 6(b) with a green line. The peaks of the radio flux were roughly coincident with the peaks of SXR derivative. Considering that the HXR flux and type III radio burst origin from the nonthermal electrons propagating downward into the chromosphere along the newly reconnected magnetic field lines and upward into the interplanetary space along the open field lines, the coincidence of the peaks during the impulsive phase of the flare implies the existence of nonthermal electrons accelerated by magnetic reconnection. Combining the positive correlation between $V_{D}$ of the CFR and SXR derivative, we conclude that the chromospheric condensation of the C3.1 flare was most probably driven by nonthermal electrons.

The investigations of chromospheric condensation in theory and numerical simulations have also made huge progress. Fisher (1986) derived an analytical expression of the condensation lifetime:

$$
\tau=\pi \sqrt{H / g}
$$

where $H$ and $g$ stand for the gravitational scale height and acceleration of gravity in the chromosphere. The typical value of $\tau$ is $\sim 1$ minute. However, superposition of continuous condensations energized at different times within an unresolved observational element naturally result in a longer condensation lifetime (Fisher 1989). In the combined modeling of acceleration, transport, and hydrodynamic response in solar flares, Rubio da Costa et al. 
(2015) found that the condensation timescale can reach $70-90 \mathrm{~s}$. In the 1D radiative hydrodynamic numerical simulations, Reep et al. (2015) found that the timescales of the electron-driven chromospheric evaporation are 3.5-4 minutes for different cases.

In Table 2, we list the timescales of chromospheric condensation and evaporation in the previous literatures. It is revealed that the timescales of evaporation upflows $(2-20$ minutes) are generally 2-3 times larger than the timescales of condensation downflows (1-7 minutes). For the C3.1 flare in this study, the condensation timescale is $3-4$ minutes, which is consistent with previous findings. To our knowledge, the cadence $(6 \mathrm{~s})$ of the IRIS raster observation is the highest ever for the investigation of chromospheric condensation.

Based on the radiative hydrodynamic flare model, Fisher (1989) derived an analytical expression of the peak downflow speed for explosive evaporation:

$$
v_{\text {peak }} \approx 0.6\left(F_{\text {evap }} / \rho_{c h}\right)^{1 / 3}
$$

where $F_{\text {evap }}$ and $\rho_{\text {ch }}$ represent the energy flux and chromospheric density. For the C3.1 flare, $v_{\text {peak }}=52 \mathrm{~km} \mathrm{~s}^{-1}$. Taking $10^{-11} \mathrm{~g} \mathrm{~cm}^{-3}$ as the typical value of $\rho_{c h}$ in the chromosphere, $F_{\text {evap }}$ is estimated to be $0.65 \times 10^{10} \mathrm{erg} \mathrm{cm}^{-2} \mathrm{~s}^{-1}$, which is close to the threshold energy flux for explosive evaporation $\left(\sim 10^{10} \mathrm{erg} \mathrm{cm}^{-2} \mathrm{~s}^{-1}\right)$. The estimated $F_{\text {evap }}$ should be a lower limit since the slit positions (S1 and S2) are located at the CFR whose maximum intensity is far less than the IFR and PFRs. According to the correlation between the intensity and $V_{D}$ of Si IV line in the left panels of Figure 10, the peak downward velocities at the IFR and PFRs should be larger and the nonthermal electron energy flux should be higher.

\subsection{What Is the Cause of QPPs?}

QPPs of solar flares have been extensively observed and reported in various wavelengths. However, the mechanism of QPPs still remains unclear. On one hand, there are diverse 
MHD waves (kink mode and sausage mode) in the solar atmosphere, such as the slow wave, fast wave, and Alfvén wave. The existence and propagation of waves may modulate the emissions in certain wavelengths (e.g. Nakariakov et al. 2004; Mariska 2005; Tan et al. 2010; Su et al. 2012a). On the other hand, the magnetic reconnection rate may be modulated by the $p$-mode wave (Chen \& Priest 2006), slow-mode wave (Nakariakov \& Zimovets 2011), Alfvén wave (Asai et al. 2001), fast sausage-mode wave (Zaitsev \& Stepanov 1982), and fast kink-mode wave (Nakariakov et al. 2006). Therefore, the acceleration and propagation of electrons in the downward and upward directions would be modulated by the periodic magnetic reconnections (Aschwanden et al. 1994; Ning et al. 2005). Besides, the tearing-mode instability and coalescence of magnetic islands occur iteratively, resulting in intermittent magnetic reconnection and particle acceleration (Kliem et al. 2000; Karlický 2004). In a single loop flare on 2005 January 1, Nakariakov et al. (2010) found QPPs of $\gamma$-ray emission with a period of $\sim 40 \mathrm{~s}$, which are also present in the HXR and microwave emissions. They proposed that the QPPs are created by periodic magnetic reconnections accompanying particle acceleration triggered by a global kink oscillation in a nearby coronal loop. In an M1.8 flare on 2002 October 20, Zimovets \& Struminsky (2010) found QPPs of both thermal and nonthermal HXR emissions with periods of $16 \mathrm{~s}$ and $36 \mathrm{~s}$, which are interpreted in terms of MHD oscillations excited in two interacting systems of flaring loops.

In our study, the QPPs with periods of $32-42 \mathrm{~s}$ are observed in UV ( $\mathrm{Si}$ IV $\lambda 1402.77$ ) and SXR derivative. As described at the beginning of Section 3.1, the length of a loop from the null point to the solar surface is estimated to be $\sim 20 \mathrm{Mm}$. If the QPPs are caused by the modulation of magnetic reconnection by fast wave propagating along the fan surface, the phase speed $\left(2 L / P_{Q P P}\right)$ is estimated to be $950-1250 \mathrm{~km} \mathrm{~s}^{-1}$, which is close to the typical value for fast wave in the corona. Hence, the QPPs in the C3.1 flare are most probably caused by the modulation of magnetic reconnection, particle acceleration, and subsequent precipitation by fast wave propagating along the fan surface loops. The nonthermal 
electrons propagating downward heat the chromosphere and generate evaporation as well as condensation. Therefore, QPPs are generated in both UV and SXR during the impulsive phase of the flare. In the X1.6 two-ribbon flare on 2014 September 10, QPPs are observed in multiwavelengths from radio to HXR (Li et al. 2015b). Our results indicate that QPPS exist not only in the typical two-ribbon flares, but also in circular-ribbon flares. The same mechanism may at work in both types.

Finally, we briefly discuss the influence of the rest wavelength on the results. As mentioned in Section 2.2, the spectral profiles of Si IV $\lambda 1402.77$ line could not been fitted by a single-Gaussian function or double-Gaussian function. Using the same method as described in Brosius \& Daw (2015), we derived the same rest wavelength of Si IV line,

i.e., $1402.86 \pm 0.0145 \AA$. Therefore, our method is reasonable and results are convincing. The blueshifted Doppler velocities of H1 (see Figure 8(d)) may be a signature of gentle chromospheric evaporation due to a very low energy flux of electrons. However, the blueshifts are too small to draw a solid conclusion.

\section{Summary}

In this paper, we report our multiwavelength observations of the C3.1 circular-ribbon flare by various instruments on 2015 October 16. The main results are summarized as follows:

1. The flare brightened first at the southwest part. As time goes on, the area of initial brightening expanded and became more complex, including several patches or kernels. Meanwhile, the narrow eastern part of flare appeared, forming the CFR that encircled the IFR. Apart from the CFR and IFR, there were a pair of short PFRs to the north of IFR, which were most striking in the IRIS/SJI $1400 \AA$ and $2796 \AA$. For the first 
time, we observe the circular-ribbon flare in the Ca II H line aboard Hinode/SOT. The shape of CFR is similar to that observed in AIA $1600 \AA$.

2. Like the $\mathrm{C} 4.2$ homologous flare, this flare was associated with positive polarities and a negative polarity inside in the AR 12434. The IFR and CFR were cospatial with the negative polarity and positive polarities, implying the existence of a magnetic null point and dome-like spine-fan topology.

3. High-cadence (6 s) IRIS raster observations show plasma downflow in the Si IV line during the impulsive phase of the flare. The Doppler velocities increased from a few $\mathrm{km} \mathrm{s}^{-1}$ to the peak values of $45-52 \mathrm{~km} \mathrm{~s}^{-1}$. Afterwards, the speeds decreased gradually to the initial levels. The timescales of the chromospheric condensation were 3-4 minutes, indicating ongoing magnetic reconnection.

4. The Doppler velocities of the condensation are positively correlated with the logarithm of the Si IV line intensities and time derivative of the SXR flux in 1-8 $\AA$. The radio dynamic spectra reveal that the flare was accompanied by a type III radio burst. Combining the correlations and radio burst, we conclude that the chromospheric condensation was most probably driven by nonthermal electrons. Using the analytical expression in Fisher (1989) and the peak downflow speed, we carried out a diagnostic of energy flux of the precipitated electrons. A lower limit of the flux is estimated to be $0.65 \times 10^{10} \mathrm{erg}^{-2} \mathrm{sm}^{-1}$.

5. During the impulsive phase of the flare, the Si IV line intensities and SXR derivative show QPPs with periods of 32-42 s. To our knowledge, this is the first report of QPPs in circular-ribbon flares. The QPPs were most probably caused by intermittent null-point magnetic reconnections modulated by the fast wave propagating along the fan surface loops at a phase speed of 950-1250 $\mathrm{km} \mathrm{s}^{-1}$. The intermittent acceleration 
and precipitations of electrons result in periodic heating observed in the Si IV line and emissions in SXR.

In the future, additional case studies using state-of-the-art, multiwavelength observations and MHD numerical simulations are required to figure out the causes of chromospheric condensation and QPPs in circular-ribbon flares.

The authors appreciate the referee for inspiring and valuable comments. We also acknowledge H. S. Ji, Y. N. Su, M. D. Ding, and L. Feng for fruitful discussions. IRIS is a NASA small explorer mission developed and operated by LMSAL with mission operations executed at NASA Ames Research Center and major contributions to downlink communications funded by the Norwegian Space Center (NSC, Norway) through an ESA PRODEX contract. SDO is a mission of NASA's Living With a Star Program. AIA and HMI data are courtesy of the NASA/SDO science teams. Hinode is a Japanese Mission, with NASA and STFC (UK) as international partners. This work is supported by NSFC (Nos. 11303101, 11333009, 11573072, 11603077), the Fund of Jiangsu Province (Nos. BK20161618, BK20161095), and the open research program of Key Laboratory of Solar Activity, National Astronomical Observatories, CAS (No. KLSA201510). 


\section{REFERENCES}

Asai, A., Shimojo, M., Isobe, H., et al. 2001, ApJ, 562, L103

Aschwanden, M. J., Benz, A. O., \& Montello, M. L. 1994, ApJ, 431, 432

Aschwanden, M. J., Benz, A. O., Dennis, B. R., \& Schwartz, R. A. 1995, ApJ, 455, 347

Battaglia, M., Kleint, L., Krucker, S., \& Graham, D. 2015, ApJ, 813, 113

Bougeret, J.-L., Kaiser, M. L., Kellogg, P. J., et al. 1995, Space Sci. Rev., 71, 231

Brosius, J. W., \& Phillips, K. J. H. 2004, ApJ, 613, 580

Brosius, J. W. 2013, ApJ, 762, 133

Brosius, J. W., \& Daw, A. N. 2015, ApJ, 810, 45

Brown, J. C. 1971, Sol. Phys., 18, 489

Carmichael, H. 1964, NASA Special Publication, 50, 451

Chen, P. F., \& Priest, E. R. 2006, Sol. Phys., 238, 313

Chen, F., \& Ding, M. D. 2010, ApJ, 724, 640

Culhane, J. L., Harra, L. K., James, A. M., et al. 2007, Sol. Phys., 243, 19

Czaykowska, A., De Pontieu, B., Alexander, D., \& Rank, G. 1999, ApJ, 521, L75

De Pontieu, B., Title, A. M., Lemen, J. R., et al. 2014, Sol. Phys., 289, 2733

Dulk, G. A., Goldman, M. V., Steinberg, J. L., \& Hoang, S. 1987, A\&A, 173, 366

Fisher, G. H., Canfield, R. C., \& McClymont, A. N. 1985, ApJ, 289, 414

Fisher, G. H., Canfield, R. C., \& McClymont, A. N. 1985, ApJ, 289, 425 
Fisher, G. H., Canfield, R. C., \& McClymont, A. N. 1985, ApJ, 289, 434

Fisher, G. H. 1986, IAU Colloq. 89: Radiation Hydrodynamics in Stars and Compact Objects, 255,53

Fisher, G. H. 1989, ApJ, 346, 1019

Fletcher, L., Hannah, I. G., Hudson, H. S., \& Innes, D. E. 2013, ApJ, 771, 104

Forbes, T. G., \& Acton, L. W. 1996, ApJ, 459, 330

Graham, D. R., \& Cauzzi, G. 2015, ApJ, 807, L22

Hayes, L. A., Gallagher, P. T., Dennis, B. R., et al. 2016, ApJ, 827, L30

Hirayama, T. 1974, Sol. Phys., 34, 323

Ichimoto, K., \& Kurokawa, H. 1984, Sol. Phys., 93, 105

Joshi, N. C., Liu, C., Sun, X., et al. 2015, ApJ, 812, 50

Karlický, M. 2004, A\&A, 417, 325

Kliem, B., Karlický, M., \& Benz, A. O. 2000, A\&A, 360, 715

Kopp, R. A., \& Pneuman, G. W. 1976, Sol. Phys., 50, 85

Kosugi, T., Matsuzaki, K., Sakao, T., et al. 2007, Sol. Phys., 243, 3

Lemen, J. R., Title, A. M., Akin, D. J., et al. 2012, Sol. Phys., 275, 17

Li, Y., \& Ding, M. D. 2011, ApJ, 727, 98

Li, D., Ning, Z. J., \& Zhang, Q. M. 2015a, ApJ, 813, 59

Li, D., Ning, Z. J., \& Zhang, Q. M. 2015b, ApJ, 807, 72 
Li, T., \& Zhang, J. 2015, ApJ, 804, L8

Mariska, J. T. 2005, ApJ, 620, L67

Masson, S., Pariat, E., Aulanier, G., \& Schrijver, C. J. 2009, ApJ, 700, 559

Milligan, R. O. 2011, ApJ, 740, 70

Nakariakov, V. M., Tsiklauri, D., Kelly, A., Arber, T. D., \& Aschwanden, M. J. 2004, A\&A, 414, L25

Nakariakov, V. M., Foullon, C., Verwichte, E., \& Young, N. P. 2006, A\&A, 452, 343

Nakariakov, V. M., \& Melnikov, V. F. 2009, Space Sci. Rev., 149, 119

Nakariakov, V. M., Foullon, C., Myagkova, I. N., \& Inglis, A. R. 2010, ApJ, 708, L47

Nakariakov, V. M., \& Zimovets, I. V. 2011, ApJ, 730, L27

Ning, Z., Ding, M. D., Wu, H. A., Xu, F. Y., \& Meng, X. 2005, A\&A, 437, 691

Ning, Z. 2014, Sol. Phys., 289, 1239

Ofman, L., \& Sui, L. 2006, ApJ, 644, L149

Pontin, D., Galsgaard, K., \& Démoulin, P. 2016, Sol. Phys., 291, 1739

Polito, V., Reeves, K. K., Del Zanna, G., Golub, L., \& Mason, H. E. 2015, ApJ, 803, 84

Polito, V., Reep, J. W., Reeves, K. K., et al. 2016, ApJ, 816, 89

Priest, E., \& Forbes, T. 2000, Magnetic reconnection: MHD theory and applications / Eric Priest, Terry Forbes. New York : Cambridge University Press, 2000

Reep, J. W., Bradshaw, S. J., \& Alexander, D. 2015, ApJ, 808, 177 
Rubio da Costa, F., Liu, W., Petrosian, V., \& Carlsson, M. 2015, ApJ, 813, 133

Sadykov, V. M., Kosovichev, A. G., Sharykin, I. N., Zimovets, I. V., \& Vargas Dominguez, S. 2016, ApJ, 828, 4

Scherrer, P. H., Schou, J., Bush, R. I., et al. 2012, Sol. Phys., 275, 207

Shibata, K., \& Magara, T. 2011, Living Reviews in Solar Physics, 8, 6

Sturrock, P. A. 1966, Nature, 211, 695

Su, J. T., Shen, Y. D., \& Liu, Y. 2012a, ApJ, 754, 43

Su, J. T., Shen, Y. D., Liu, Y., Liu, Y., \& Mao, X. J. 2012b, ApJ, 755, 113

Sych, R., Nakariakov, V. M., Karlicky, M., \& Anfinogentov, S. 2009, A\&A, 505, 791

Tan, B., Zhang, Y., Tan, C., \& Liu, Y. 2010, ApJ, 723, 25

Teriaca, L., Falchi, A., Cauzzi, G., et al. 2003, ApJ, 588, 596

Tian, H., Li, G., Reeves, K. K., et al. 2014, ApJ, 797, L14

Tian, H., Young, P. R., Reeves, K. K., et al. 2015, ApJ, 811, 139

Torrence, C., \& Compo, G. P. 1998, Bulletin of the American Meteorological Society, 79, 61

Tsuneta, S., Ichimoto, K., Katsukawa, Y., et al. 2008, Sol. Phys., 249, 167

Wang, T. J., Ofman, L., \& Davila, J. M. 2009, ApJ, 696, 1448

Young, P. R., Doschek, G. A., Warren, H. P., \& Hara, H. 2013, ApJ, 766, 127

Young, P. R., Tian, H., \& Jaeggli, S. 2015, ApJ, 799, 218

Zaitsev, V. V., \& Stepanov, A. V. 1982, Soviet Astronomy Letters, 8, 132 
Zhang, Q. M., Chen, P. F., Guo, Y., Fang, C., \& Ding, M. D. 2012, ApJ, 746, 19

Zhang, Q. M., Ning, Z. J., Guo, Y., et al. 2015, ApJ, 805, 4

Zhang, Q. M., Li, D., Ning, Z. J., et al. 2016, ApJ, 827, 27

Zimovets, I. V., \& Struminsky, A. B. 2010, Sol. Phys., 263, 163 
Table 1: Description of the observational parameters.

\begin{tabular}{lcccc}
\hline \hline Instrument & $\lambda$ & Time & Cadence & Pixel Size \\
& $(\AA)$ & $(\mathrm{UT})$ & $(\mathrm{s})$ & $($ arcsecs $)$ \\
\hline SDO/AIA & $94-335$ & $10: 10: 00-10: 30: 00$ & 12 & 0.6 \\
SDO/AIA & 1600,1700 & $10: 10: 00-10: 30: 00$ & 24 & 0.6 \\
SDO/HMI & 6173 & $10: 10: 00-10: 30: 00$ & 45 & 0.5 \\
IRIS/SJI & 1400,2796 & $10: 15: 15-10: 36: 10$ & 12.6 & 0.333 \\
IRIS/raster & Si IV 1402.77 & $10: 15: 15-10: 20: 32$ & 6 & 0.333 \\
Hinode/SOT & Ca II H 3968 & $10: 00: 33-10: 38: 33$ & 120 & 0.218 \\
GOES & $0.5-4,1-8$ & $10: 10: 00-10: 50: 00$ & 2.04 & - \\
WIND/WAVES & $0.02-13.825 \mathrm{MHz}$ & $10: 10: 00-10: 50: 00$ & 60 & - \\
KRIM & $250-350 \mathrm{MHz}$ & $10: 16: 00-10: 31: 00$ & 0.25 & - \\
BLENSW & $10-80 \mathrm{MHz}$ & $10: 15: 00-10: 30: 00$ & 0.25 & - \\
\hline
\end{tabular}


Table 2: List of the timescales ( $\tau$ in minute) of chromospheric condensation and evaporation in previous literatures.

\begin{tabular}{lcccc}
\hline \hline Author & Flare class & Date & Type & $\tau$ \\
\hline Ichimoto \& Kurokawa (1984) & 1B & 1982 & condensation & $\leq 1$ \\
Czavkowska et al. (1999) & M6.8 & $1998 / 04 / 29$ & condensation & $\sim 2.5$ \\
Brosius \& Phillips (2004) & M2.0 & $2001 / 04 / 24$ & condensation & $\sim 3$ \\
Graham \& Cauzzi (2015) & X1.6 & $2014 / 09 / 10$ & condensation & $\sim 1$ \\
Sadvkov et al. (2016) & M1.0 & $2014 / 06 / 12$ & condensation & $\sim 7$ \\
\hline Brosius (2013) & C1.0 & $2012 / 03 / 07$ & evaporation & $\sim 2$ \\
Graham \& Cauzzi (2015) & X1.6 & $2014 / 09 / 10$ & evaporation & $\sim 12$ \\
Battaglia et al. (2015) & X1.0 & $2014 / 03 / 29$ & evaporation & $\sim 5$ \\
Polito et al. $(2015)$ & C6.5 & $2014 / 02 / 03$ & evaporation & $\sim 6$ \\
Polito et al. $(\underline{\underline{2016}})$ & X2.0 & $2014 / 10 / 27$ & evaporation & $\sim 13$ \\
$\underline{\text { Sadvkov et al. (2016) }}$ & M1.0 & $2014 / 06 / 12$ & evaporation & $\sim 20$ \\
\hline
\end{tabular}




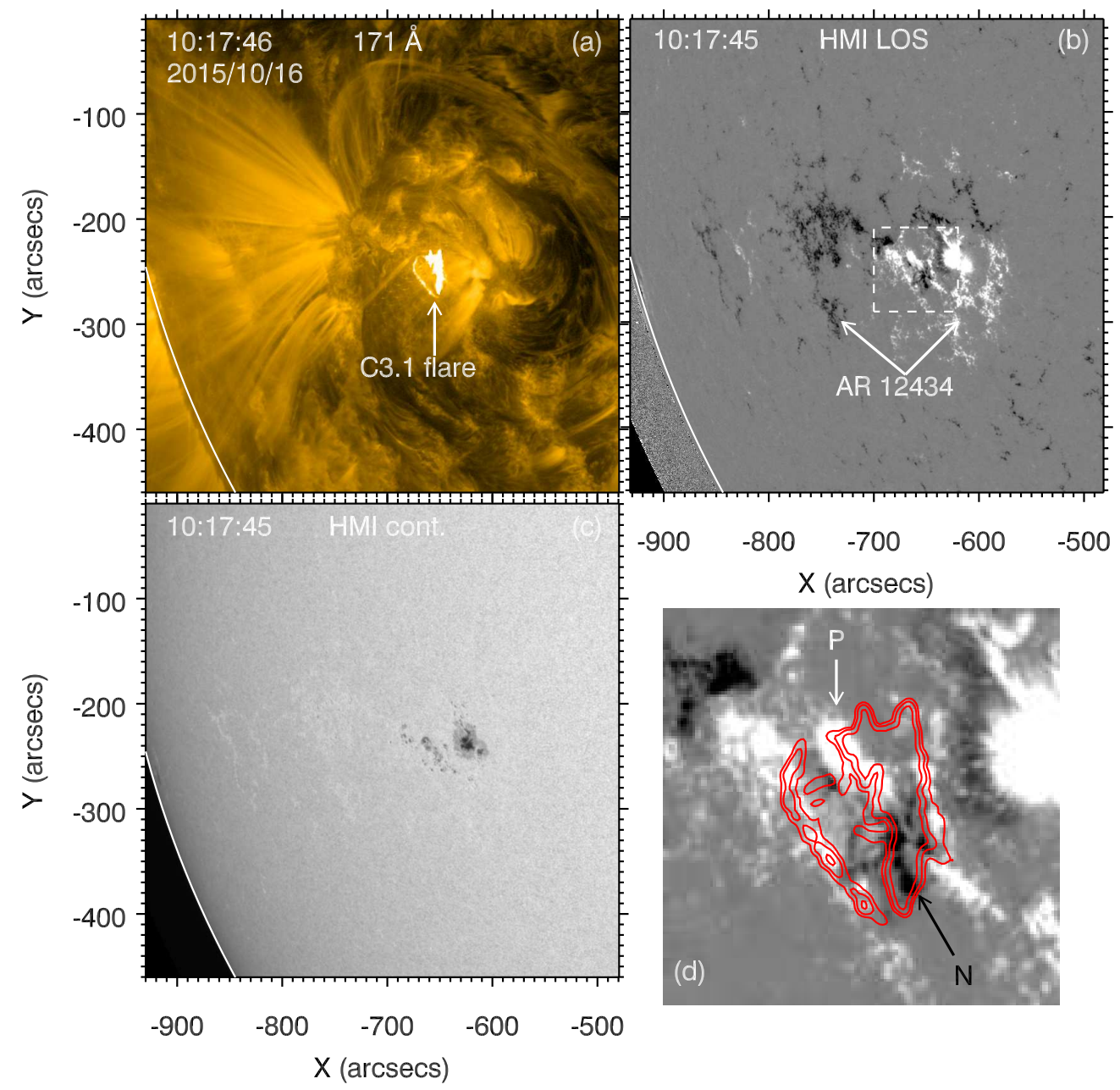

Fig. 1.- (a) AIA $171 \AA$ image at 10:17:46 UT. The white arrow points to the C3.1 cicularribbon flare. (b)-(c) HMI LOS magnetogram and white-light continuum image at 10:17:45 UT. The white arrows point to the main negative and positive polarities of AR 12434. (d) A close-up of the flare region within the white dashed box of panel (b). The intensity contours of the $171 \AA$ image in panel (a) are overlaid with red lines. 


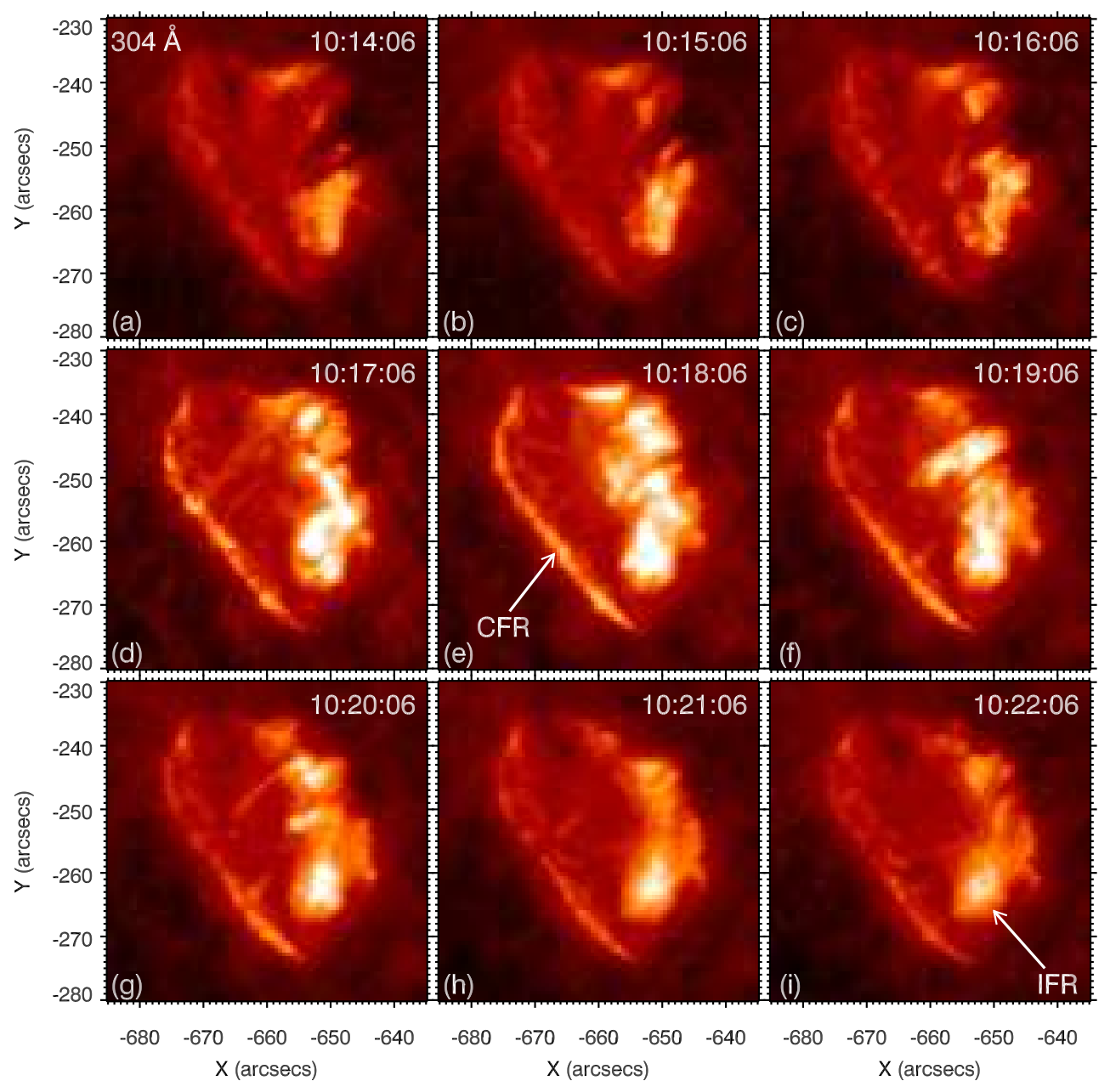

Fig. 2.- Snapshots of the AIA $304 \AA$ images. In panels (e) and (i), the white arrows point to the CFR and IFR, respectively.

(Animations of this figure are available in the online journal.) 

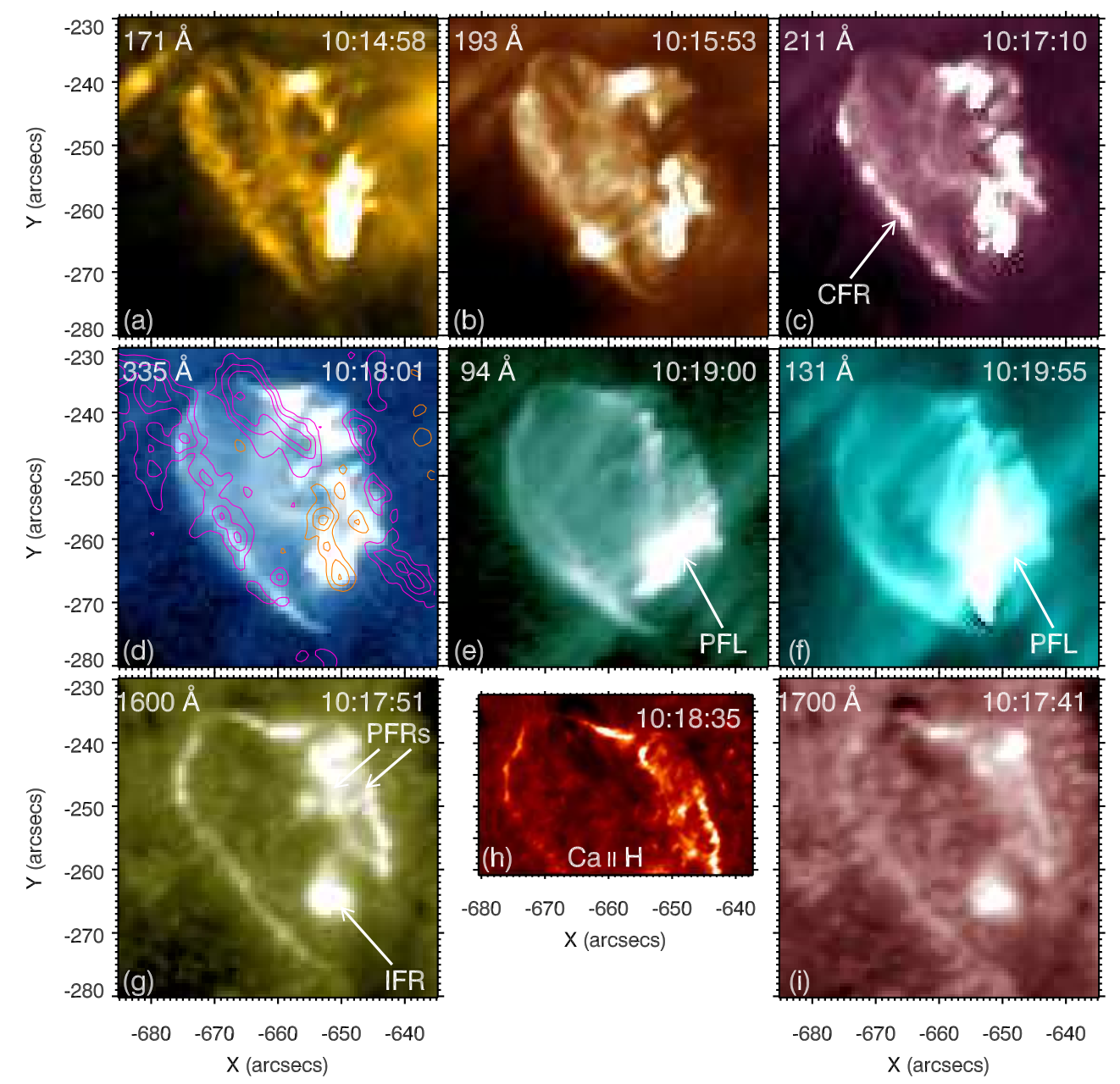

Fig. 3.- Snapshots of the AIA EUV and UV images and SOT Ca II H image. In panels (c) and (g), the white arrows point to the CFR, IFR, and PFRs. In panel (d), the magenta and orange lines stand for the contours of the positive and negative LOS magnetic fields, respectively. In panels (e) and (f), the white arrows point to the bright PFL connecting the PFRs.

(Animations of this figure are available in the online journal.) 


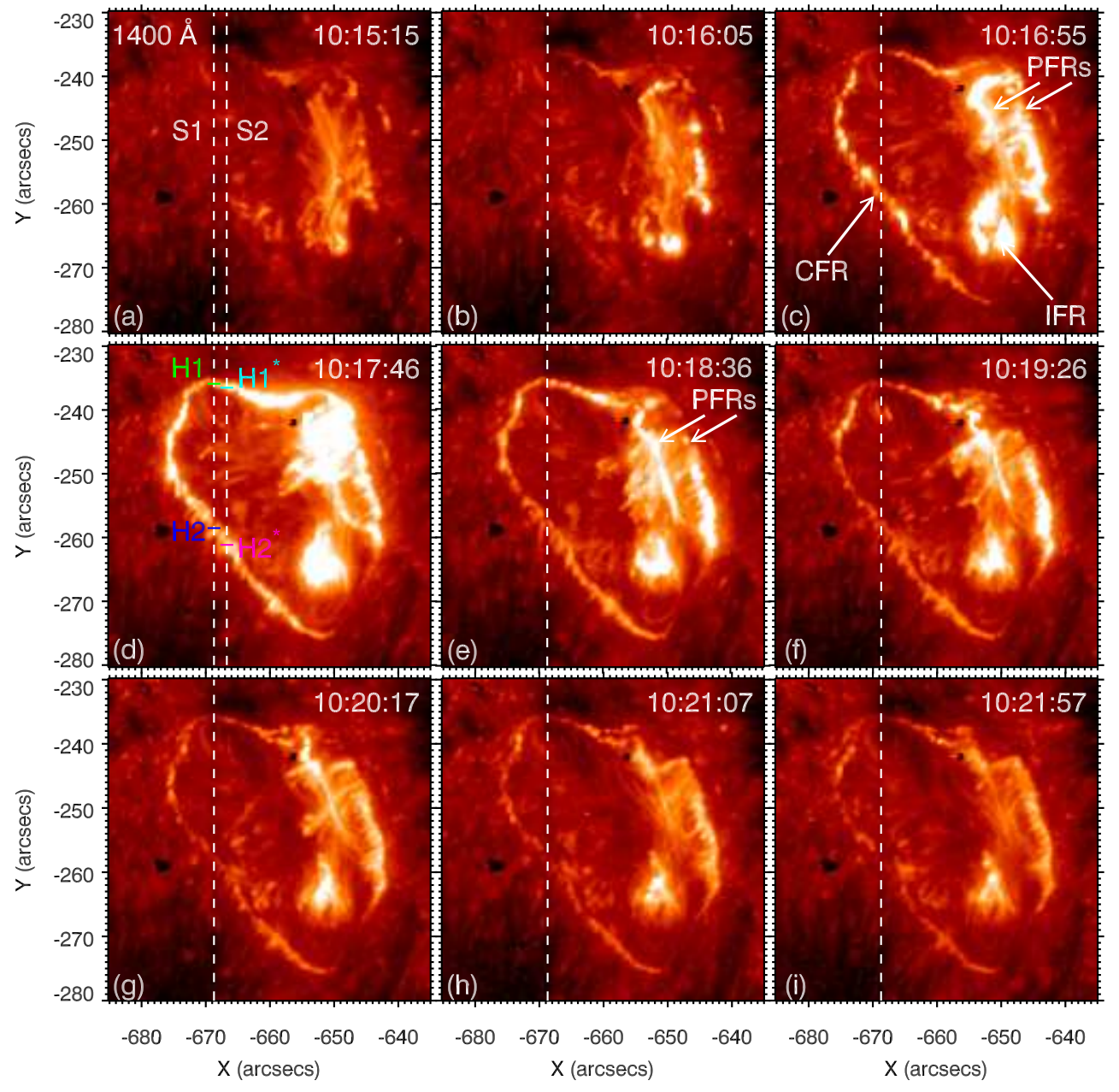

Fig. 4.- Snapshots of the IRIS/SJI $1400 \AA$ images. In panel (a), the two vertical dashed lines denote the slit positions of the two-step raster $(\mathrm{S} 1, \mathrm{~S} 2)$. In panel (c), the white arrows point to the CFR, IFR, and PFRs. In panel (d), S1 encounters the CFR at H1 and H2. S2 encounters the CFR at $\mathrm{H}^{*}$ and $\mathrm{H}_{2}^{*}$.

(Animations of this figure are available in the online journal.) 


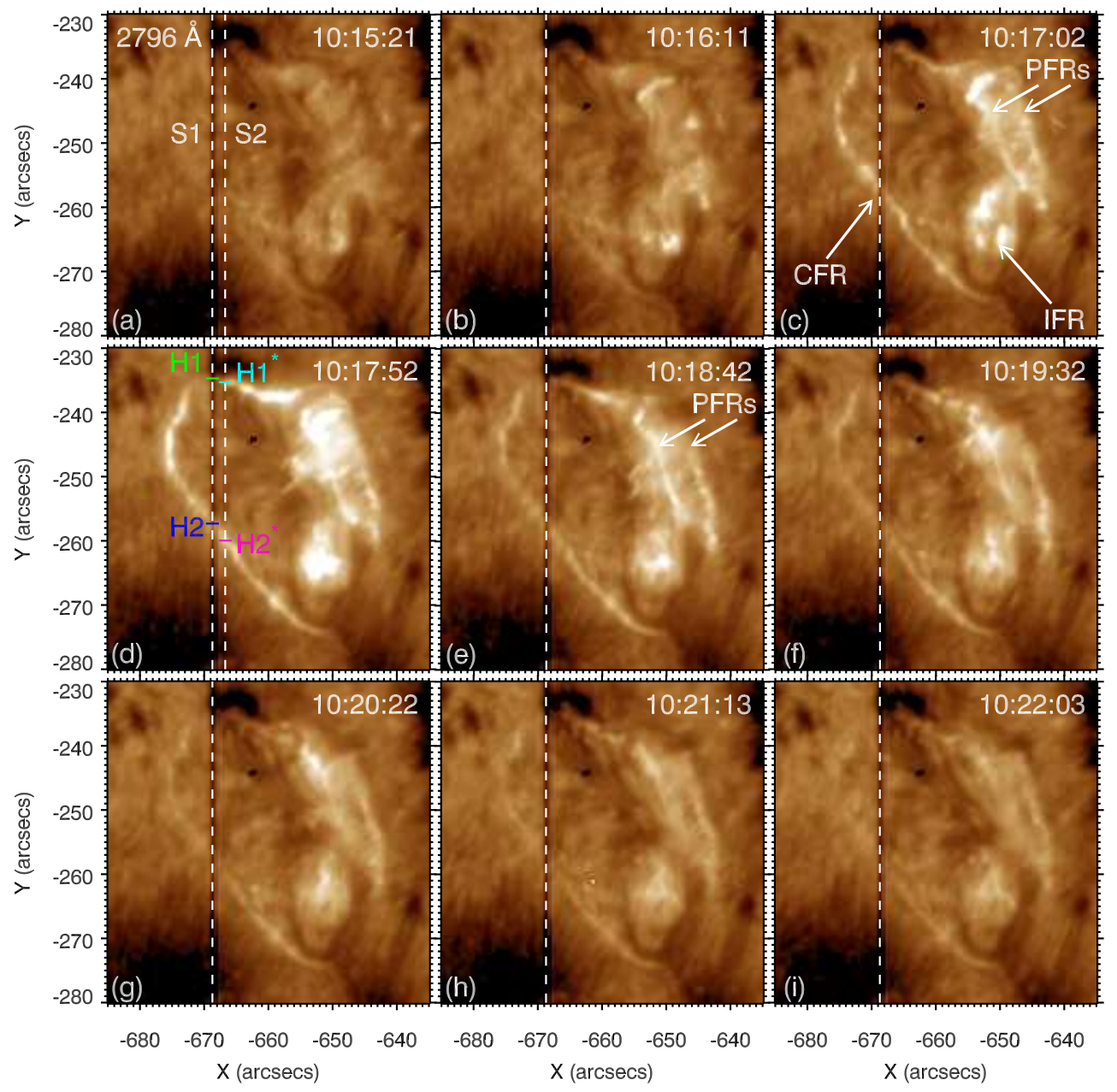

Fig. 5.- Same as Figure 4, but for IRIS/SJI $2796 \AA$. 


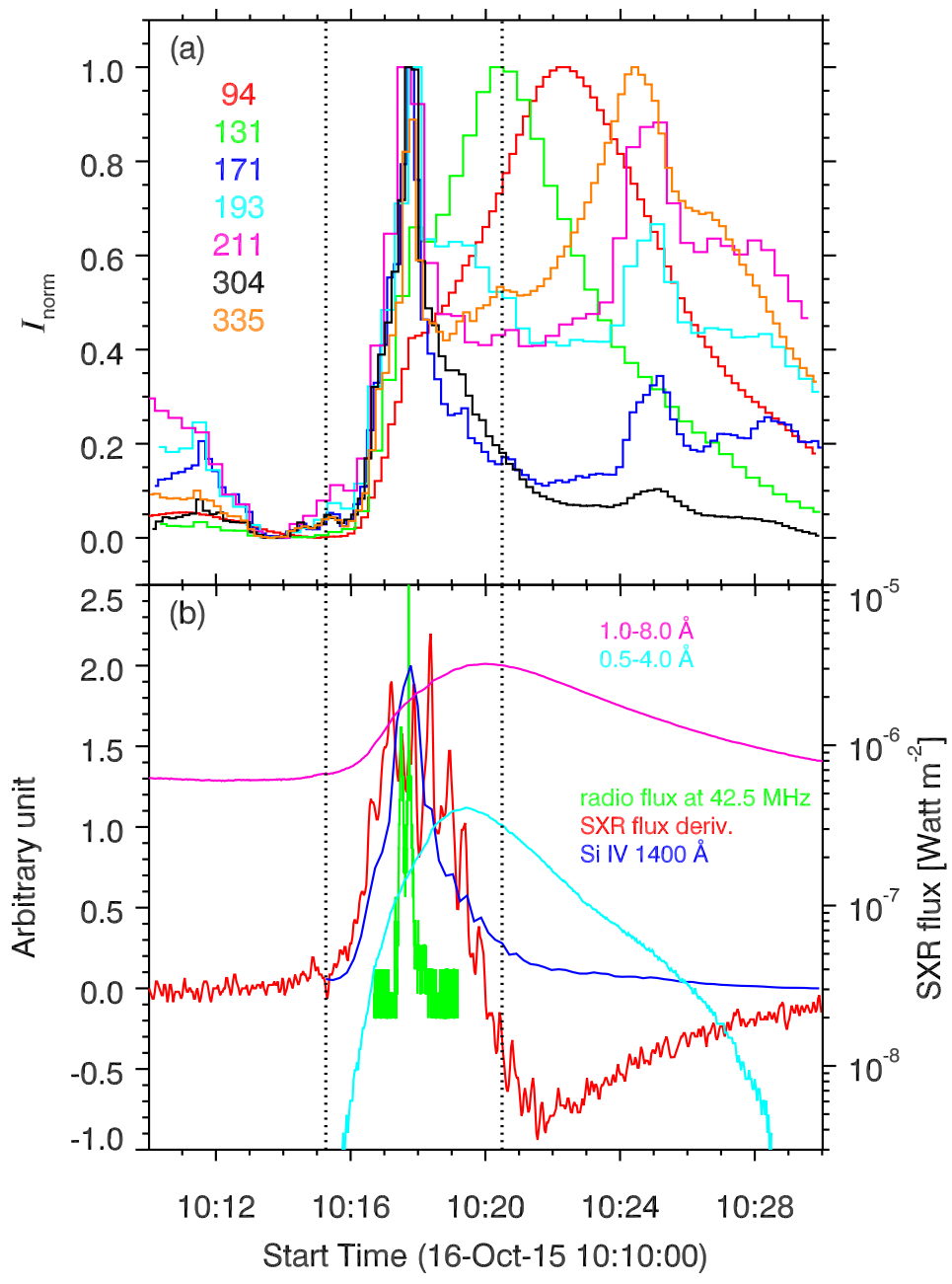

Fig. 6.- (a) Normalized EUV light curves of the flare during 10:10-10:30 UT. (b) SXR light curves of the flare during 10:10-10:30 UT in 0.5-4 $\AA$ (cyan) and 1-8 $\AA$ (magenta). The time derivative of the flux in $1-8 \AA$ is drawn with a red line. The light curve of the radio flux at $42.5 \mathrm{MHz}$ is drawn with a green line. The light curve of the flare in $1400 \AA$ is illustrated with a blue line. The two dotted lines denote the starting (10:15:15 UT) and ending (10:20:32 UT) times of the IRIS raster observation in each panel. 

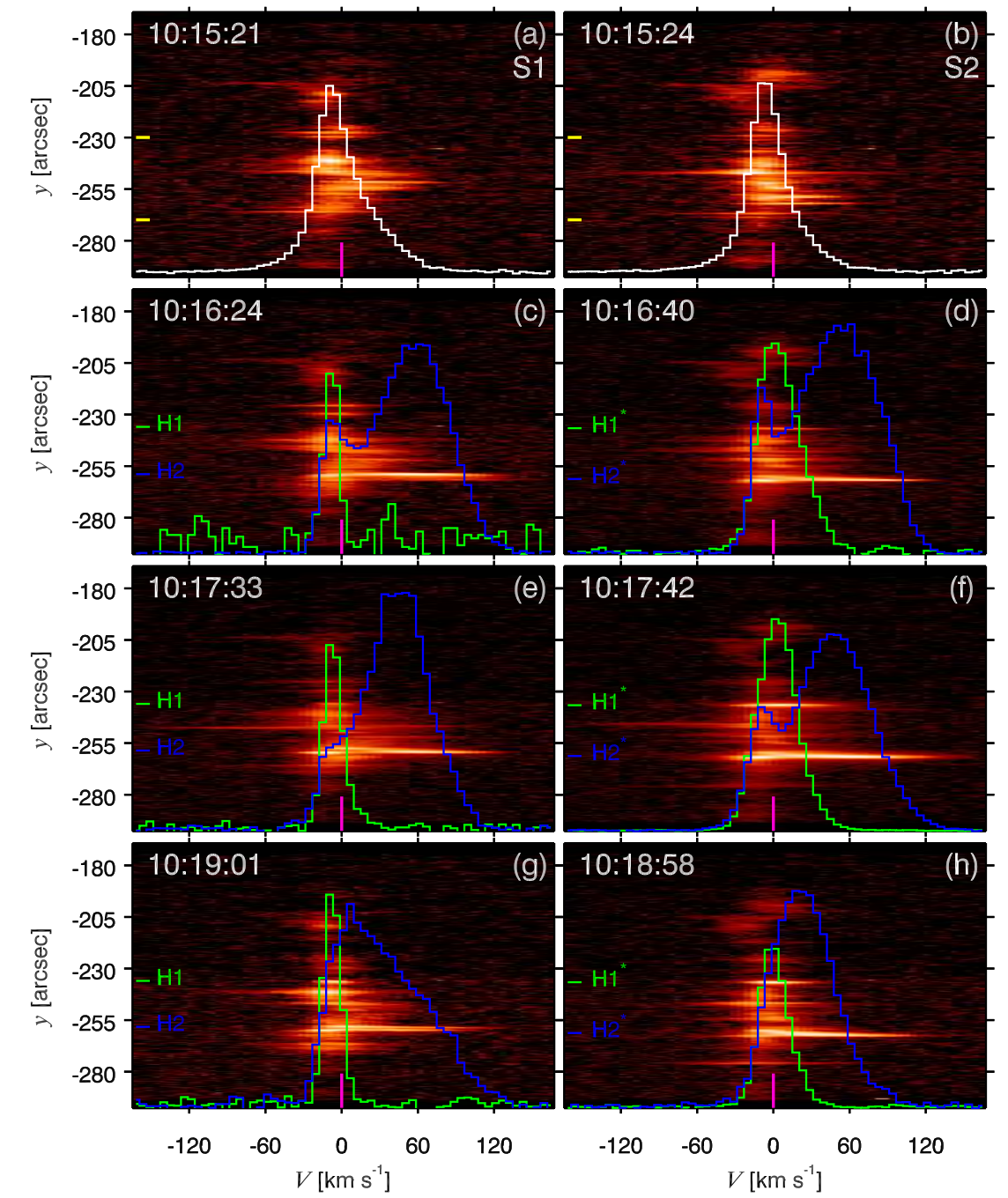

Fig. 7.- IRIS spectral window of Si IV for S1 (left panels) and S2 (right panels). In panels (a) and (b), the white curves represent the spacetime average spectra between the positions $\left(-270^{\prime \prime}\right.$ and $\left.-230^{\prime \prime}\right)$ marked by the two yellow lines during 10:15:21-10:15:43 UT. The rest wavelength $\left(\lambda_{0}\right)$ of Si IV $\lambda 1402.77$ is calculated to be $1402.86 \pm 0.0145 \AA$. In panels (c)-(h), the green curves are the spectra at $\mathrm{H} 1$ and $\mathrm{H} 1 *$ in three raster scans. The blue curves are the spectra at $\mathrm{H} 2$ and $\mathrm{H} 2 *$ in three raster scans. 

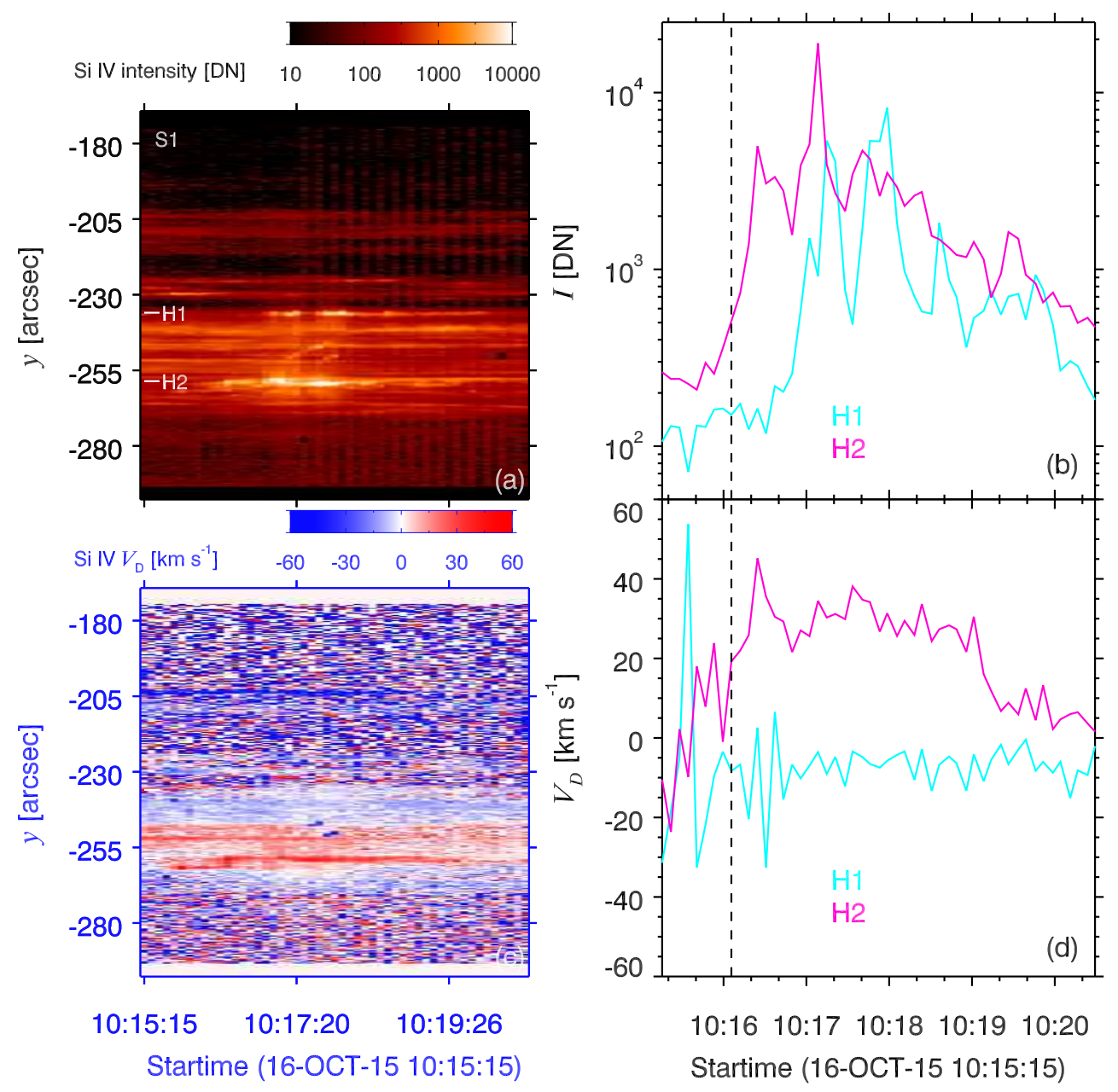

Fig. 8.- (a, c) Temporal evolutions of the Si IV line intensity and Doppler velocity along slit S1. (b, d) Temporal evolutions of the intensity and Doppler velocity of H1 and H2. The dashed line denotes the starting time for performing correlation analysis in Figure 10, 

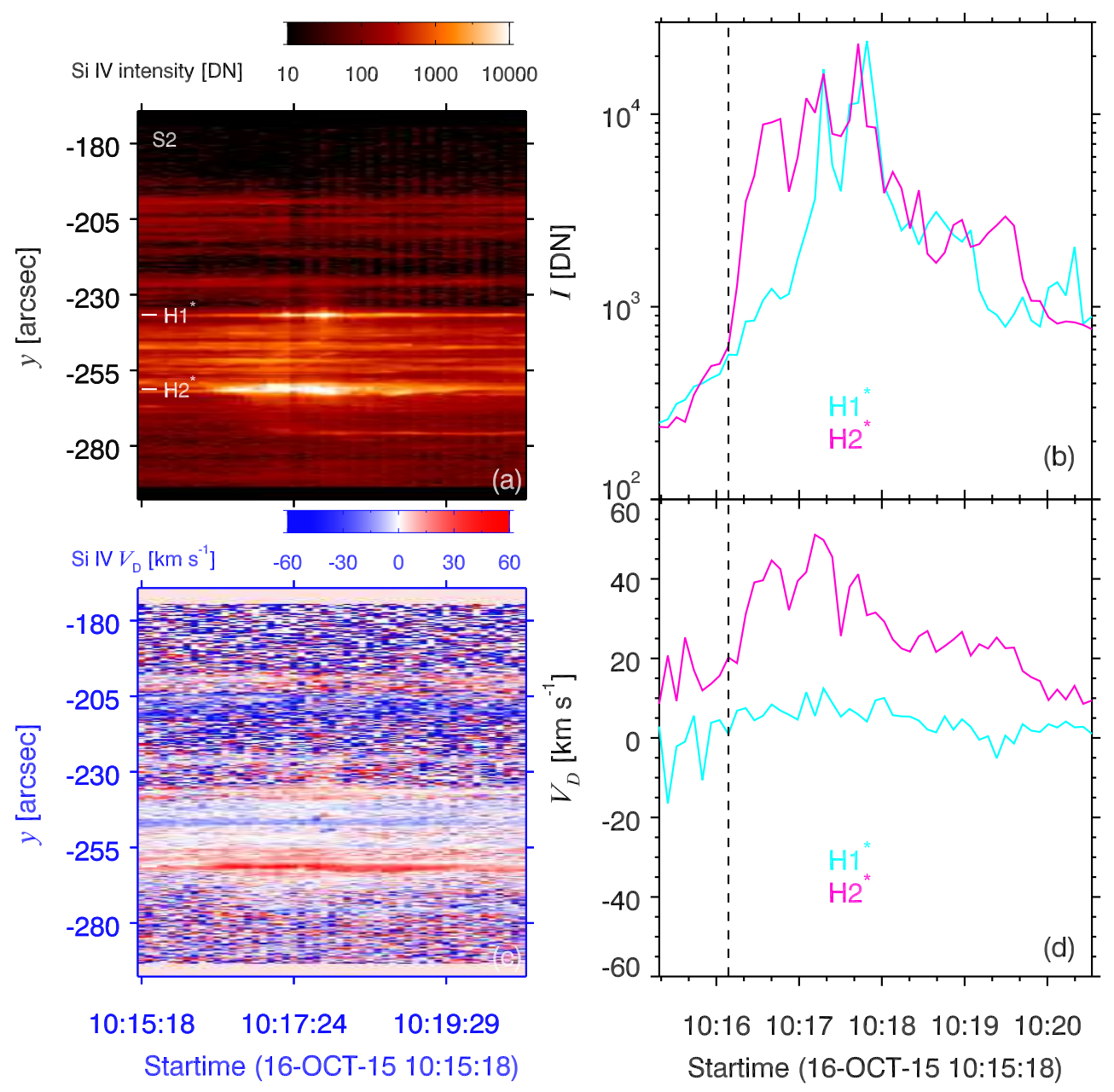

Fig. 9.- (a, c) Temporal evolutions of the Si IV line intensity and Doppler velocity along slit S2. (b, d) Temporal evolutions of the intensity and Doppler velocity of H1* and H2*. The dashed line denotes the starting time for performing correlation analysis in Figure 10. 


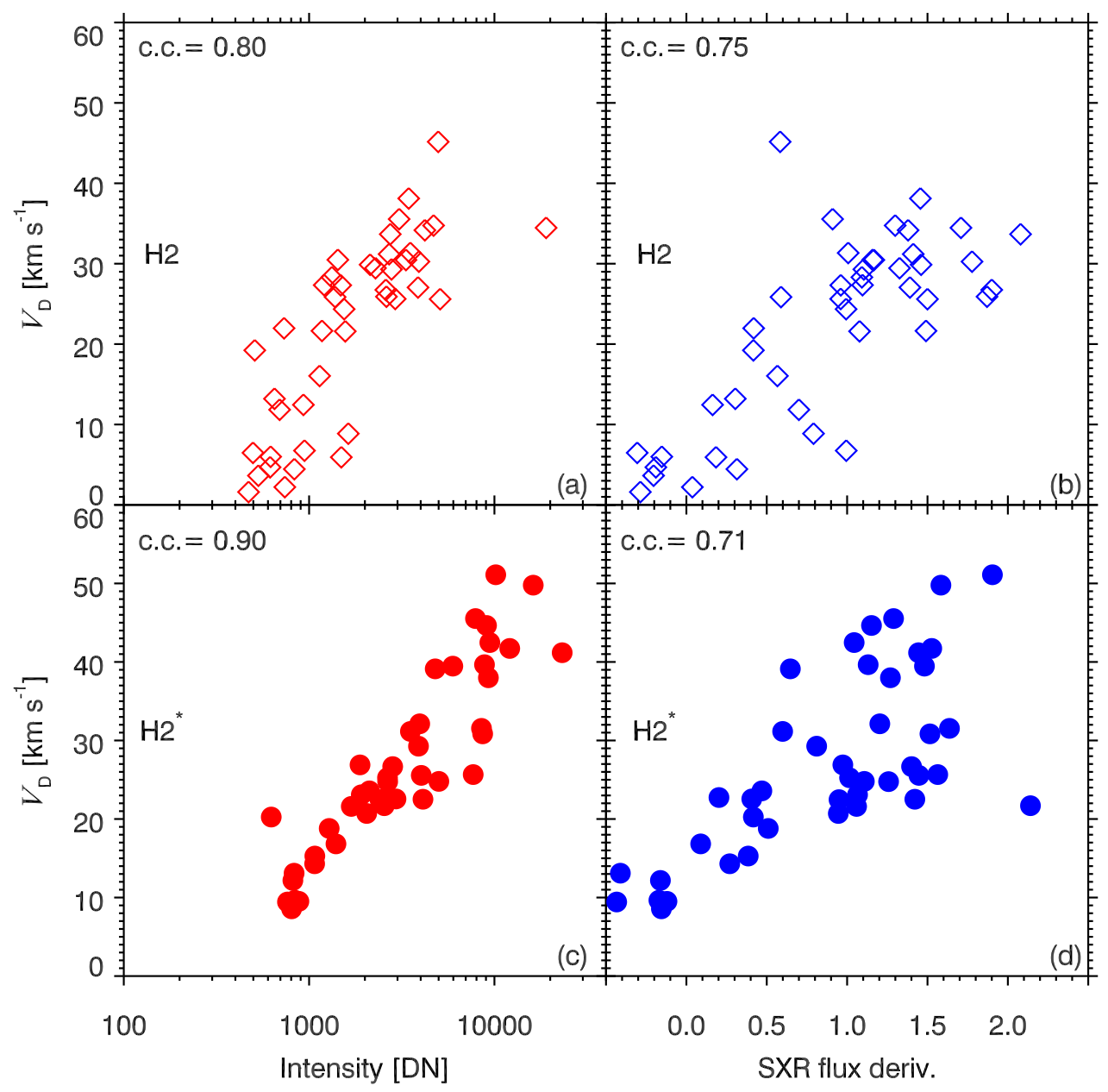

Fig. 10.- Scatter plots of Si IV Doppler velocity and intensity (left panels) and SXR flux deriviative (right panels) for $\mathrm{H} 2$ (top panels) and $\mathrm{H} 2 *$ (bottom panels). Note the intensities are in log-scale. The correlation coefficients are also displayed in each panel. 

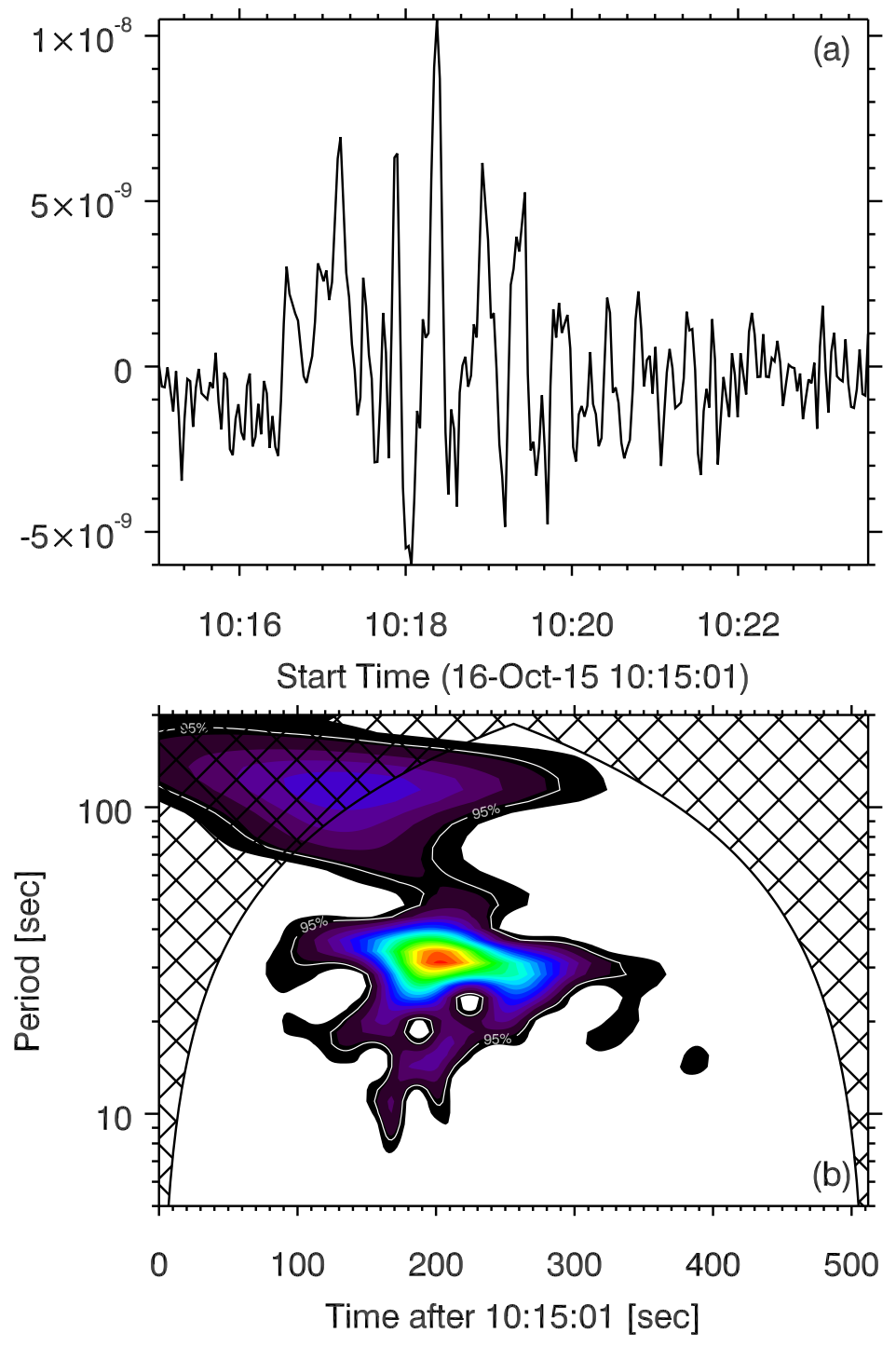

Fig. 11.- (a) Fast-varying component of the time derivative of SXR flux in Figure 6(b). (b) Morlet wavelet transform of the component. The red color represents the highest power. The white, solid contour encloses regions of $\geq 95 \%$ confidence level for a white noise process (Torrence \& Compo 1998). The cross-hatched regions indicate the "cone of influence", where edge effects become important (Wang et al. 2009). 


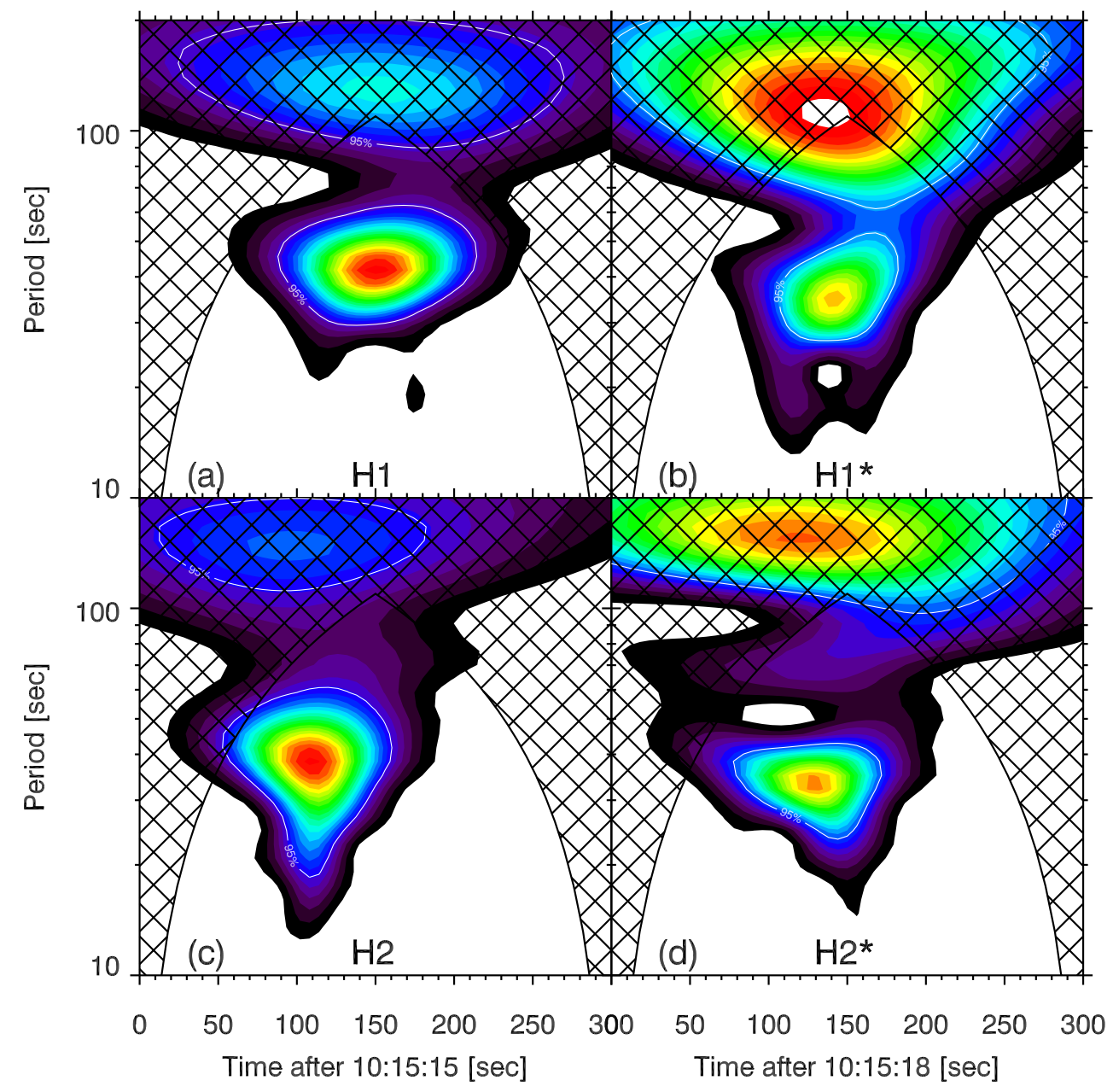

Fig. 12.- Morlet wavelet transforms of the fast-varying components of the Si IV intensities of $\mathrm{H} 1$ (a), H1* (b), H2 (c), and H2* (d). The annotations have the same meanings as in Figure 11(b). 

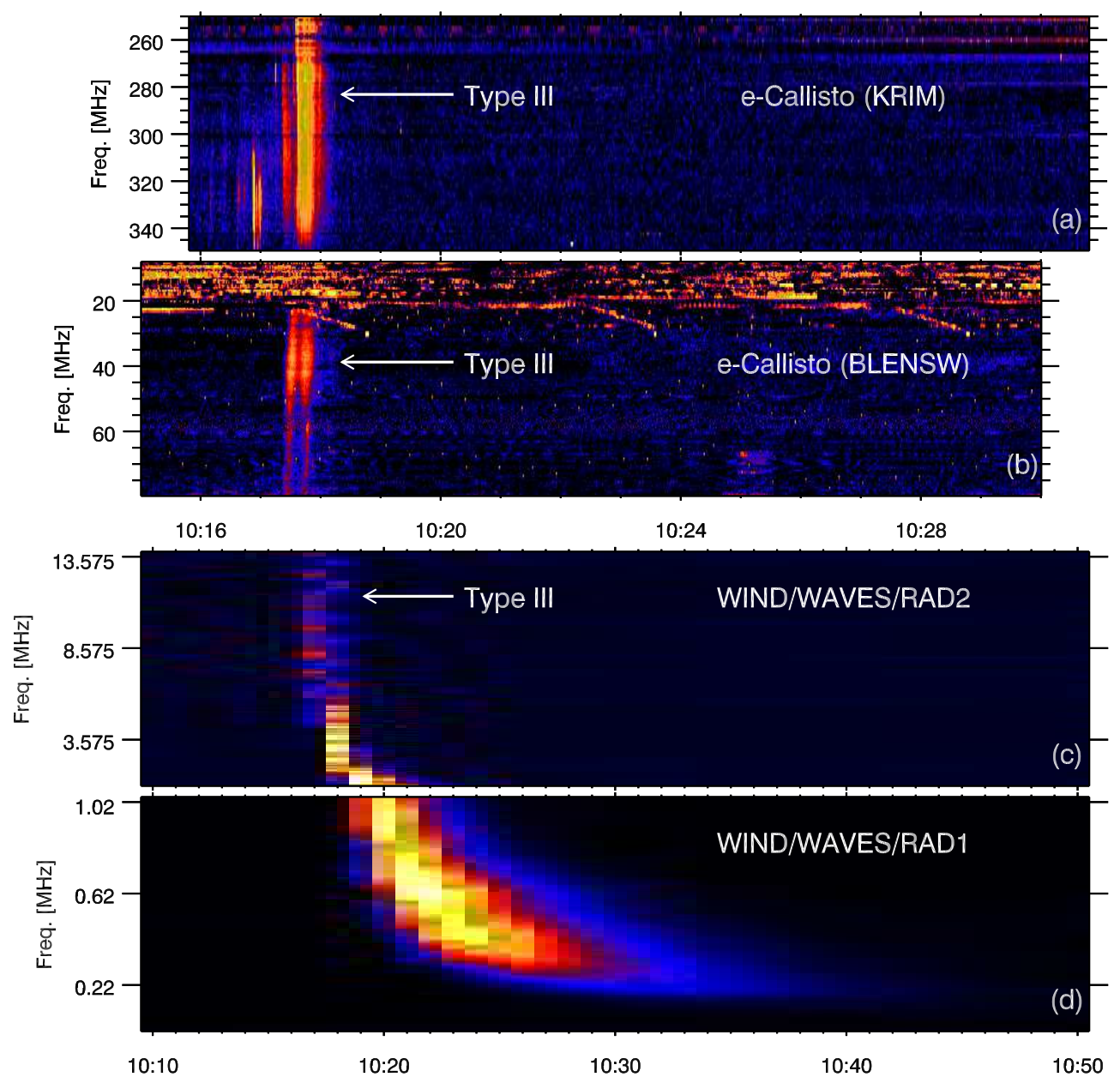

Fig. 13.- Radio dynamic spectra recorded by $\operatorname{KRIM}$ (a), BLENSW (b), and WIND/WAVES (c-d). The time is in UT and the frequency is in MHz. The arrows point to the type III radio burst with enhanced emissions and fast frequency drift rate. 